\title{
Revisión taxonómica del género Molineriella Rouy (Gramineae: Poeae: Airinae) en la Península Ibérica
}

\author{
Jesús Cebrino Cruz \& Carlos Romero-Zarco \\ Departamento de Biología Vegetal y Ecología. Facultad de Biología. Universidad de Sevilla. C/ Profesor García \\ González s/n. 41012 Sevilla, España.
}

\section{Correspondencia \\ C. Romero-Zarco \\ e-mail: zarco@us.es}

Recibido: 11 septiembre 2017

Aceptado: 26 octubre 2017

Publicado on-line: diciembre 2017

\begin{abstract}
Resumen
Se presenta una revisión del género Molineriella Rouy sobre la base del estudio morfológico de 1.300 ejemplares de herbario. Se trata de un género mediterráneo que tiene su máxima riqueza específica en la Península Ibérica, y para el que se han reconocido aquí tres especies, para las cuales se aportan descripciones y mapas de distribución en el área de estudio. Se han designado un neotipo para Aira laevis Brot. y sendos lectotipos para Periballia minuta subsp. australis Paunero y Molineria minuta var. baetica Willk. Se ha descrito como nueva Molineriella laevis var. submutica Cebrino \& Romero Zarco.
\end{abstract}

Palabras clave: Gramineae, Poeae, Airinae, Molineriella, Periballia, Península Ibérica, Taxonomía, Tipificación.

\begin{abstract}
Taxonomic revision of the genus Molineriella Rouy (Gramineae: Poeae: Airinae) in the Iberian Península

We present the revision of the genus Molineriella Rouy based on a morphological study of 1,300 herbarium specimens. It is a Mediterranean genus with its maximum species richness in the Iberian Peninsula. Three species are recognized, described and maped relating to the study area. A neotypus for Aira laevis Brot. and lectotypi for Periballia minor subsp. australis Paunero, and Molineria minuta var. baetica Willk. have been designated. Molineriella laevis var. submutica Cebrino \& Romero Zarco has been described as new.
\end{abstract}

Key words. Gramineae, Poeae, Airinae, Molineriella, Periballia, Iberian Peninsula, Taxonomy, Typification.

\section{Introducción}

La preparación de la síntesis taxonómica del género Molineriella Rouy para el volumen XIX de Flora iberica ha permitido estudiar con detalle este pequeño pero interesante género que actualmente se incluye en la subtribu Airinae Fr. de la tribu
Poeae R. Br. (cf. Soreng et al., 2010 en adelante), junto con Aira L., Periballia Trin., Corynephorus P. Beauv., Antinoria Parl., Avenella Drejer y Helictochloa Romero Zarco, todos ellos exclusivos del Viejo Mundo (Eurasia y $\mathrm{N}$ de África).

Linneo reunió en Aira un conjunto heterogéneo de gramíneas caracterizadas por sus espiguillas

Este trabajo ha sido financiado con los proyectos del Ministerio de Economía y Competitividad Flora iberica IX(2) y X(2), CGL2012-32914 y CGL2015-66161-P, respetivamente, cofinanciados con fondos FEDER, en el grupo de investigación RNM-204 del Plan Andaluz de Investigación (Junta de Andalucía, España). 
bifloras, con dos glumas semejantes (Linneo, 1754: $31)$. De las 14 especies descritas en la primera edición de Species Plantarum (Linneo, 1753: 6366) solo dos, Aira praecox L. y Aira caryophyllea L., permanecen clasificadas en el género Aira en su actual delimitación, y una sola de sus especies, Aira minuta L., se incluye en el género Molineriella en la actualidad. Nuevas especies fueron descritas durante los siglos XVIII y XIX. Entre ellas son de especial interés para este estudio Aira involucrata Cav., A. laevis Brot., A. glabrata Brot. y A. lendigera Lag. Estas especies fueron clasificadas por diversos autores en los géneros Airopsis Desv., Catabrosa P. Beauv., Periballia Trin., Molineria Parl. o Molineriella Rouy, dando origen a un cierto número de combinaciones nomenclaturales.

Varios autores del siglo XX, siguiendo el criterio de Ascherson y Graebner (1899), han mantenido un concepto amplio del género Periballia, incluyendo a las especies que en este estudio se separan en el género Molineriella, criterio aceptado en las principales recopilaciones genéricas publicadas a nivel mundial (Clayton \& Renvoize, 1986; Watson \& Dallwitz, 1992), así como en la monografía de Paunero (1957). Sin embargo, Paunero (1964), en base al estudio de la anatomía foliar y de otros caracteres anatómicos de las especies españolas (que son todas las conocidas hasta la fecha), defendió la separación de dos géneros: Periballia [P. involucrata (Cav.) Trin.] y Molineria [incluyendo M. minuta (L.) Parl., M. australis (Paunero) Paunero y Molineria laevis (Brot.) Hack.]. Previamente, Rouy (1913) había puesto de manifiesto la ilegitimidad de Molineria Parl. por ser homónimo posterior de Molineria Colla (Hypoxidaceae), publicando en su lugar un nuevo nombre: Molineriella Rouy, con Molineriella minuta (L.) Rouy como tipo implícito. La separación de los géneros Periballia y Molineriella ha sido aceptada en varios estudios florísticos (Tutin, 1980; Romero-
Zarco, 1987; Devesa, 1995; Bolòs \& Vigo, 2001; Romero, 2011; Tison \& Foucault, 2014). Este criterio está apoyado por los resultados del estudio filogenético de Döring (2009), quien encontró, con marcadores moleculares cloroplásticos, que Periballia y especies de los géneros Avenella Drejer, Corynephorus P. Beauv. y Aira, foman un grupo diferente del compuesto por las especies de Molineriella, las cuales están más relacionadas con Helictotrichon subg. Pratavenastrum (Vierh.) Holub (actualmente el género Helictochloa Romero Zarco). Aunque las relaciones filéticas del género Molineriella lo relacionan con el género Helictochloa, morfológicamente Molineriella se parece más a Aira o Peribalia que a Helictochloa.

Los datos cariológicos disponibles (Albers, 1980; Chromosome Counts Database, version $1.45<$ <ttp://ccdb.tau.ac.il>; IPCN Chromosome Reports <http://www.tropicos.org/Project/IPCN>, consultado el 25 de septiembre de 2017) muestran un número básico de cromosomas $x=7$ para los géneros Aira y Periballia; sin embargo, en las especies del género Molineriella la mayoría de los recuentos cromosómicos indican un número básico $x=4$, con algunos recuentos discrepantes para $M$. minuta y $M$. laevis.

La frecuencia de materiales de herbario mal identificados o confundidos con especies del género Aira justifica la necesidad de ofrecer una información más detallada sobre la taxonomía y variabilidad del género.

\section{Material y métodos}

Para el estudio morfométrico se han utilizado aproximadamente 1.300 pliegos procedentes de los 21 herbarios siguientes (según Thiers, 2017): $\mathrm{ABH}, \mathrm{BC}, \mathrm{COI}$, ELVE, FCO, G, GDA, HVR, JACA, LEB, LISE, LISI, LISU, MA, MGC, SALA, SANT, SEV, UEVH, UNEX y VAL.

Caracteres mofológicos estudiados

Porte (altura de la planta completa)

Tallo (excluida la inflorescencia)

Diámetro del tallo

Longitud de las hojas (limbo)

Anchura de las hojas (limbo)

Lígulas superiores e inferiores

Longitud de la panícula

Número de ramificaciones de la panícula

Longitud de la espiguilla

Gluma superior e inferior
Longitud del lema

Longitud de la pálea

Arista

Callo (forma y longitud)

Pelos del callo (presencia y longitud)

Longitud de las anteras

Lodículas

Longitud del fruto

Diámetro del fruto

Tabla 1. Caracteres morfológicos estudiados. Table 1. Morphological characters studied. 
Los caracteres estudiados se relacionan en la tabla 1. Las medidas de las espiguillas y de las piezas espiculares se realizaron en una lupa binocular LEICA S6E (hasta 40 aumentos), utilizando una regleta sólida pautada en décimas de milímetro con una precisión subjetiva aproximada de 0,05 $\mathrm{mm}$. Para el estudio detallado de la morfología del callo, se utilizó un microscopio electrónico Pro-x PW-100-011 (part nr), 800-07333 (model nr). Los mapas de distribución se realizaron mediante el programa ArcGIS ver. 9.3.1. (ArcGIS9, ESRI Inc, Redlands, CA, USA; ArcGIS9 2009).

\section{Resultados}

\section{Delimitación morfológica del género}

Del género Aira, en el que inicialmente fueron descritas sus especies, Molineriella difiere por una relación diferente entre la longitud de las glumas y de los lemas, así como la presencia y posición de la arista. En Molineriella, los lemas alcanzan o superan los extremos de las glumas, son obtusodenticulados, no aristados o con una arista recta o flexionada, que nace en tercio superior del dorso del lema. Por el contrario, en las especies de Aira los lemas quedan ocultos por las glumas, son bífidos, con dos dientes agudos en el ápice y, en la mayoría de las especies, llevan una arista claramente geniculada que nace en el tercio inferior del dorso, al menos en la flor apical de la espiguilla.

Periballia involucrata se reconoce entre las especies de la subtribu Airinae por la presencia de uno o más verticilos de ramas estériles en los nudos inferiores de la panícula. A este caracter morfológico y a otros anatómicos destacados por Paunero (1964), se añade aquí la diferencia en la morfología del pedúnculo de la espiguilla. En $P$. involucrata, el pedúnculo presenta un engrosamiento apical anguloso que alcanza aproximadamente el doble de la anchura de la parte media, mientras que en las especies del género Molineriella el engrosamiento apical del pedúnculo no es anguloso y alcanza al menos el triple de la anchura de la zona media.

\section{Caracteres taxonómicos}

En la tabla 1 se relacionan los caracteres analizados en el material de herbario estudiado, comentándose a continuación los de mayor interés taxonómico.

Porte. Todas las especies son anules, unicaules o multicaules, con raíz fibrosa. Molineriella minuta reúne las plantas de menor porte dentro del género, que no sobrepasan por lo general los 17 cm (incluyendo a la inflorescencia), mientras que en $M$. laevis y $M$. australis llegan a alcanzar hasta $48 \mathrm{~cm}$. En las descripciones, las medidas de los tallos excluyen la inflorescencia.

Longitud del lema. Los lemas varían de 1,1 a 2,3 mm de longitud. Los tamaños menores de 1,5 $\mathrm{mm}$ corresponden solo a $M$. minuta, y los valores de 1,5 mm o superiores a las otras dos especies.

Callo. El callo puede ser glabro o más o menos peloso. En M. laevis y $M$. minuta, el callo puede ser casi enteramente glabro o presentar pelos relativamente cortos, rectos y rígidos, hasta de $0,5 \mathrm{~mm}$, mientras que en $M$. australis siempre hay pelos relativamente largos $[(0,2) 0,4-1 \mathrm{~mm}]$, más finos, ondulados y menos rígidos (fig. 1).

Arista. Los lemas son por lo general aristados
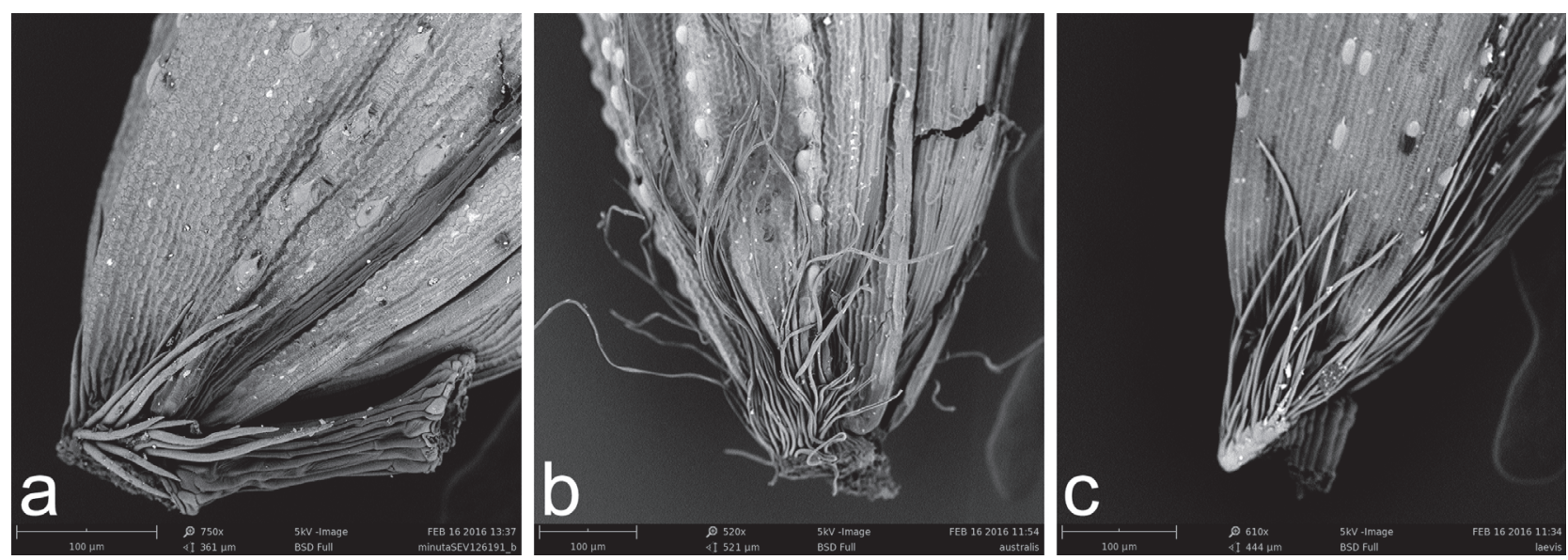

Figura 1. Detalle de la base del lema (callo) en las especies del género Molineriella, a) M. minuta, Pozoblanco, río Guadarramilla, Córdoba (SEV 126191); b) M. australis, Fuenteovejuna, Córdoba (SEV 109933); c) M. laevis var. laevis, Villanueva de los Castillejos, Huelva (SEV 151784). Figure 1. Details of the lemma base (callus) in Molineriella species. 
en $M$. laevis, y no aristados en las restantes especies, aunque de manera excepcional se han encontrado ejemplares de $M$. laevis con aristas muy reducidas o incluso ausentes (véase la variabilidad de la especie más adelante) (Fig. 2).

Anteras. La longitud de las anteras está posiblemente relacionada con el sistema de reproducción. Molineriella minuta presenta anteras pequeñas, de 0,2-0,3 $\mathrm{mm}$, que a menudo quedan atrapadas entre el lema y la pálea en antesis, lo que probablemente facilite la autofecundación, mientras que las otras dos especies presentan anteras mayores, de 0,6-1,2 $\mathrm{mm}$ y claramente exertas en antesis, síndrome compatible con un sistema de reproducción más abierto.

\section{Tratamiento sistemático}

Molineriella Rouy, FI. France 14: 102 (1913)

Molineria Parl., FI. Ital. 1: 236 (1850), nomen illeg., non Colla (1826)

Hierbas anuales. Hojas con vaina de márgenes libres; lígula membranácea; limbo plano o enrollado. Inflorescencia en panícula ramosa, laxa; ramas y pedúnculos flexuosos, divaricados, lisos, con pulvínulos bien desarrollados; pedúnculos clavados. Espiguillas pedunculadas, ligeramente comprimidas lateralmente, con 2 flores hermafroditas; pedúnculo claviforme, con un engrosamiento apical de diámetro aproximadamente el triple del diámetro de la zona media, redondeado, no anguloso. Glumas 2 , subiguales o ligeramente desiguales, la inferior algo más corta que la superior, ovadas o elípticas, subagudas, anchamente membranáceas; la inferior uninervada, la superior trinervada, con los nervios laterales muy cortos. Raquilla articulada, no prolongada por encima de la flor superior, glabra. Flores sobrepasando algo a las glumas, semejantes entre sí, ambas articuladas con la raquilla. Lema obovado, oblongo o elíptico, truncado o irregularmente dentado, membranáceo, aristado o no, escabriúsculo, con 5-7 nervios prominentes; callo obtuso, casi glabro o peloso; arista, cuando existe, recta, rara vez flexionada,

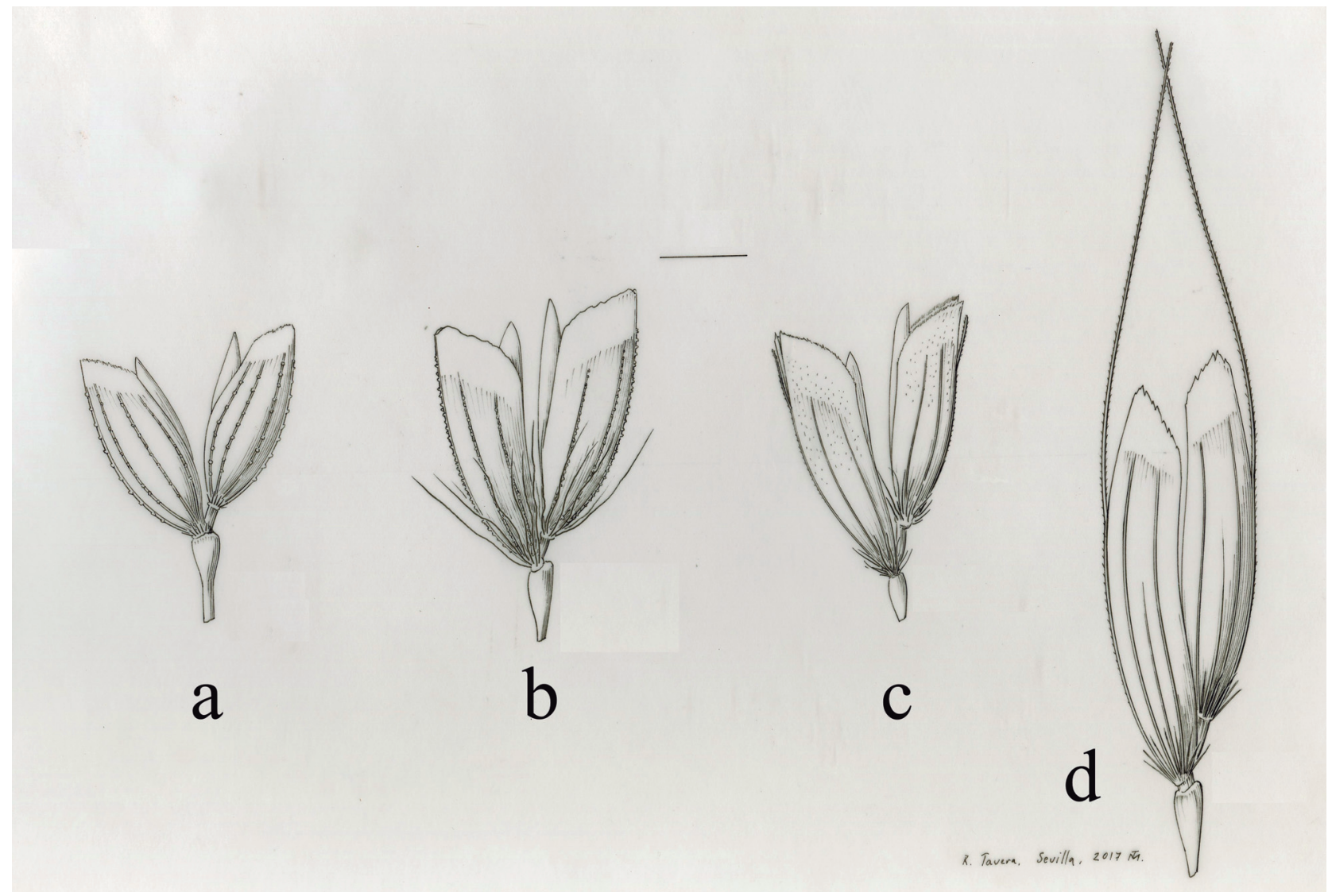

Figura 2. Espiguillas del género Molineriella desprovistas de las glumas, a) M. minuta, carretera de Villanueva del Duque a Estación de Belalcázar, Córdoba (SEV 102439); b) M. australis, entre Sanlúcar la Mayor y Aznalcázar, Sevilla (SEV 102442); c) M. laevis var. submutica, Crato, Alto Alentejo (LISI 53783/1999); d) M. laevis var. laevis, Villanueva de los Castillejos, Huelva (SEV 151784). Figure 2. Spikelets of the genus Molineriella without the glumes. 
dorsal, que nace en el tercio superior. Pálea algo más corta que el lema, biaquillada, hialina, con las quillas cilioladas. Lodículas 2, lanceoladas, con un diente lateral. Estambres 3. Ovario glabro. Fruto oblongo-elipsoideo, ligeramente comprimido, libre; hilo puntiforme.

Especie tipo: Molineriella minuta (L.) Rouy [ $\equiv$ Molineria minuta (L.) Parl.]. En aplicación del artículo 7.4 del CIB el tipo de Molineriella debe ser el mismo que el de Molineria Parl., quien solo incluyó esta única especie (Parlatore, 1850: 237).

\section{Clave para las especies}

1. Lema 1,2-2(2,5) $\mathrm{mm}$, oblongo, casi siempre aristado, excepcionalmente sin arista; callo con pelos de 0,1$0,5 \mathrm{~mm}$, rectos ................................. 3. M. laevis

- Lema 1-1,8 mm, ovado o elíptico, sin arista; callo glabro o con pelos de 0,1-0,3 $\mathrm{mm}$, rectos y rígidos, o bien de $(0,2) 0,4-1 \mathrm{~mm}$, ondulados ....................... 2

2. Lema 1-1,3 mm; anteras 0,1-0,3 mm; callo glabro o con algunos pelos de 0,1-0,3 $\mathrm{mm}$, rectos $\mathrm{y}$ rígidos ........................................ 1. M. minuta

- Lema 1-1,8 mm; anteras 0,7-1 mm; callo con pelos de $(0,2) 0,4-1 \mathrm{~mm}$, ondulados

2. M. australis

1. M. minuta (L.) Rouy, FI. Fr. 14: 102 (1913)

Aira minuta L., Sp. PI. 64 (1753) [basiónimo]

$\equiv$ Molineria minuta (L.) Parl., Fl. Ital. 1: 236 (1850)

= Periballia minuta (L.) Asch. \& Graebn., Syn.

Mitteleur. FI. 2(1): 298 (1899)

Indicación locotípica: "Habitat in Hispania"

[lectotipo designado por H. Scholz in Taxon 49: 244

(2000): Löfling s. n., LINN 85.4].

Ilustraciones: J. A. Devesa, Las Gramíneas de Extremadura: 127 lám. XXVII a-b (1991); E.

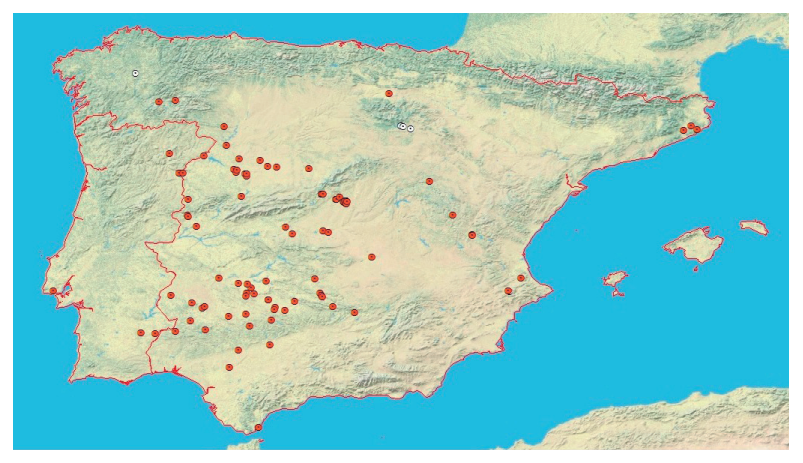

Figura 3. Mapa de distibución de Molineriella minuta en la Península Ibérica: material estudiado (círculos oscuros) y localidades adicionales tomadas de la bibliografía (círculos claros). Figure 3. Distribution map of Molineriella minuta in the Iberian Peninsula: studied material (dark circles), and additional localities from the bibliography (white circles).
Paunero in Anales Inst. Bot. Cavanilles 14: 228 figs. a, c-i, k, m (1957) [sub Periballia minuta subsp. minuta]; Valdés \& al., Fl. Andalucía Occid. 3: 329 (1987) [sub M. minuta subsp. minuta]; fig. 2a.

Hierba anual, de 2,3-16,5 cm, uni- o multicaule. Tallos de 1,8-13 cm de longitud y 0,2-0,6 mm de diámetro, lisos o ligeramente acostillados. Hojas glabras, con vaina acostillada; lígula subaguda, la de las hojas inferiores de $(0,5) 0,7-1,5 \mathrm{~mm}$, y la de las hojas superiores de 1-2,5 mm; limbo 5-57 $\times 0,4-1,2 \mathrm{~mm}$, plano o enrrollado. Inflorescencia 0,5-4 cm, hasta con 4 órdenes de ramificación. Espiguillas 1,1-1,6 mm; pedúnculo 1,2-5 mm. Glumas 1-1,4 mm, subiguales 0 la inferior ligeramente más corta que la superior, ovadas o elípticas, subobtusas, variegadas, verdosas en la parte inferior, violáceas en el centro y hialinas hacia los bordes. Artejo de la raquilla c. 0,2 mm, glabro. Lema 1-1,3 mm, ovado, sin arista, con 5-7 nervios prominentes y escabriúsculos, variegado $\mathrm{o}$ de un pardo amarillento; callo 0,1-0,2 mm, orbicular, obtuso, glabro o con algunos pelos de 0,1-0,3 mm, rectos. Pálea 0,8-1,1 mm, algo más corta que el lema. Lodículas 0,3-0,4 mm. Anteras 0,1-0,3 mm. Fruto $0,7-1 \times 0,3-0,5 \mathrm{~mm}$.

Número cromosómico: $n=9$ (Afzal-Rafii \& Zevaco, 1974); $2 n=8$ (Devesa et al., 1990a); 14 (Kozhuharuv \& Petrova, 1991).

Ecología, fenología y distribución

Pastizales efímeros, principalmente en substratos arenosos de carácter ácido; 40-1.100 m s.n.m. II-V(VI). S de Europa, Anatolia y NW de África; introducida en Australia. Dispersa por casi toda la Península Ibérica salvo el extremo $\mathrm{N}$ y el SE (fig. 3).

Observaciones. A las localidades que se relacionan más abajo habría que añadir otras de las provincias de Alicante (Serra et al., 2012), Lugo (Seijas, 1952) y Soria (Segura et al. 1998). En el Herbario del Jardín Botánico de Madrid se conserva un pliego (MA 162117) con varios ejemplares de $M$. minuta que se separaron en la época de E. Paunero de otro pliego antiguo de Clemente procedente de Gibraltar (MA 7454) que contiene un ejemplar multicaule de $M$. australis. No existe ninguna otra evidencia de que $M$. minuta esté presente en la provincia de Cádiz. Es posible que al tratarse de un pliego antiguo se reunieran en él plantas de distinta procedencia, por lo que no damos credibilidad a la localidad que se indica en la etiqueta mientras no se pueda confirmar por otros medios. Del Herbario de la Facultad de Farmacia de Madrid se ha estudiado un pliego recolectado por González Fragoso en mayo de 1882, presuntamente en Dos Hermanas (Sevilla), que contiene una mezcla de tres especies: $M$. 
minuta, M. australis y M. laevis (MAF 25994), de las cuales solo se puede confirmar la veracidad de la localidad para la segunda especie. Es probable que se hayan mezclado en dicho pliego plantas de distinta procedencia. El número cromosómico gamético $n=9$, indicado por Afzal-Rafii \& Zevaco (1974) en mitosis polínica con material de Cerdeña, difiere notablemente del los números básicos conocidos en la tribu (Albers, 1980) y del número esporofítico $2 n=8$ encontrado por Devesa et al. (1990a) en material de Badajoz. El número cromosómico $2 n=14$ dado por Kozhuharuv \& Petrova (1991) para plantas de Bulgaria requiere confirmación.

\section{Selección del material estudiado}

ESPAÑA. Ávila. Madrigal de las Altas Torres, 6-IV-2003, C. Aedo (MA 698706). Badajoz. CastueraMalpartida, 11-III-1965, P. Montserrat \& J. M. Gómez (JACA 2765). Fregenal de la Sierra, 27-VII-1988, G. Fernández \& F. Vázquez (MGC 33918). Cáceres. Coria, 23-II-1978, E. Rico (MA211289). Sierra de Gata, VI-1904, M. Gandoger (COI 79027). Ciudad Real. Alcázar de San Juan, IV-1895, ex Caroli Pau Herbarium hispanicum (MA 7462). Almodóvar del Campo, finca Pedroche, 1-IV-1993, P. Montserrat (JACA 4193). Valle de Alcudia, 9-IV-1979, M. Ladero \& Daniel (GDA 8739). Córdoba. Belalcázar, carretera hacia la Estación del Zújar en intersección con un arroyo, 20-III-1986, M. Jiménez (FCO 13839). Pozoblanco, río Guadarramilla, 13-IV-1982, A. Aparicio et al. (SEV 126191). Sierra Morena, sin fecha ni recolector (MA 7448). Cuenca. Saceda-Trasierra, Sierra de Altamira (VAL 97464). Talayuelas (VAL 73797). Gerona. Bajo Ampurdán, Santa Cristina d'Aro, 8-IV-1948, Font Quer (BC 106188). Cassá de la Selva, 17-IV-1919, Font Quer, BC 78173. Entre Sils y Maçanet de la Selva, IV1985, L. Villar (BC 675667). Huelva. Aroche, junto a orilla del río Chanza, 15-III-1998, E. Sánchez Gullón (SEV 154309). Arroyomolinos de León, dehesas de La Gitana, 9-IV-1963, P. Montserrat \& M. Capdevila (JACA 142063). Jaén. Santa Elena, 9-V-1918, C. Vicioso (MA 7468). La Rioja. Cidamón, 9-IV-1983, J. A. Alejandre (MA 452004). León. Cembranos (SALA 6945). Marne (SALA 5106). Madrid. El Escorial, IV-1873, M. Laguna (MA 7456). La Moncloa, 2-IV-1946, Rivas [sic] (SANT 2346). Monte EI Pardo, 22-IV-1917, C. Vicioso (MA 7459). Orense. Cerca de Candeda, sin fecha, S. Ortiz (SANT 33968). Río Bibei, Pueblo de Trives, 14-VI-1958, Bellot \& Casaseca (SANT 9877). Salamanca. Almenara de Tormes, 3-IV-1978, J. Sánchez (MA 292056). Florida de Liébana, linde con Villaselva y Barregas, 11-IV-1962, P. Montserrat (JACA 11362). Guijuelo, 25-III-1988, E. Rico \& J. Serradilla (MA 476607). Segovia. Santa María la Real de Nieva, 30-III1985, T. Romero (MA 566367). Sevilla. Tocina, IV-1882, González Fragoso (MAF 25997). Toledo. San Bartolomé de las Abiertas, río Sangrera, 11-III-1990, J. L. Castillo \& R. Cordero (MA 515371). Talavera de la Reina, 18II-1978, A. Segura Zubizarreta, (MA 362699). Valencia. Bocairent, Pla d'Aparisi, 18-IV-1988, J.R. Nebot (VAL 63381). Quatretonda, La Vall d'Albaida 13-V-2000, A. Conca \& J.E. Oltra (VAL 146082). Zamora. Bercianos de Valverde 23-IV-1991, J. M. Velasco (SALA 58784).
Castrillo de la Guareña 30-IV-1983, X. Giráldez (SALA 30727). Fermoselle, 30-III-1983, A. Guillén et al. (SALA 43654).

PORTUGAL. Baixo Alentejo. Colina de Santufo, Serpa, V-1882, J. Daveau (COI 78524). Quinta de Pandula, norte de Serpa, III-1881, J. Daveau (LISU 4495). Vila Verde de Ficalho, III-1880, J. Daveau (LISU 4497). Estremadura. Queluz, IV-1851, Welwitsch (G 00035039). Trás-os-Montes e Alto Douro. Quinta da Terejinha, Bragança, 28-III-1943, PI. A. de Barro Carneiro (COI 78525). Vila Flor, Santa Comba da Vilariça, 20-042001, A. L. Crespí et al. (HVR 12487).

2. M. australis (Paunero) E. Rico in Anales Jardín Bot. Madrid 38: 184 (1981)

Periballia minuta subsp. australis Paunero in Anales Inst. Bot. Cavanilles 14: 200 (1957) [basiónimo]

三 Molineria australis (Paunero) Paunero in Anales Inst. Bot. Cavanilles 21: 345 (1964)

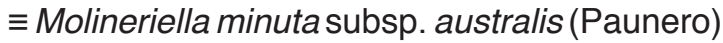

Rivas Martínez in Lazaroa 2: 168 (1980)

- Aira lagascae sensu Kunze, Chloris Austro Hispanica: 55 (1846), non Kunth (1833-1835)

= Aira lendigera var. mutica Boiss., Voy. Bot. Espagne 2: 650 (1844) [lectotipo designado por Burdet et al. (1981: 557): "Alvinas del Alcornoque / Serranias / Mr Haenseler 1839" (G, foto!)]

= Molineria minuta var. baetica Willk., Prodr. Fl. Hispan. 1: 63 (1861) [lectotipo, designado aquí: "H.M. Willkommii iter hispanicum. // Catabrosa minuta Trin. b baetica. / (Aira minuta Löffl.) pl. exsicc. $\mathrm{N}^{\circ} 561$. // Hab. in arenosis umbrosis regionis calidis: In pi- / netis inter oppida Chiclana et Conil. // Altitudo: - // Nom. vulgare: - // Legi die 19 mensis Martii 1845." (COI-Willkomm 00036125, foto!); isolectotipos: BM 001134068, BM 001134068, fotos!]

= Periballia minuta var. baetica (Willk.) Font Quer in Cavanillesia 7: 72 (1935)

Indicación locotípica: "Gibraltar (Clemente, MA, núm. 7454); Chiclana (Cádiz) (Gros, MA, núm. 7445); Córdoba (Sagredo, MA, núm. 7447); El Pedroso (Sevilla) (Gz. Fragoso, MA, núm.7444); Dos Hermanas (Sevilla) (Gz. Fragoso, MA, núm. 7446, MAF); Esparragosa de Lares (Badajoz) (Gz. Guerrero, MA, núm.7442); Badajoz (Sennen, MA, núm.7443); Alvinas del Alcornoque; $S^{a}$ de Ronda (Boissier, G.); Chiclana y Conil (Cádiz) (Willkomm, G.); Medina Sidonia (Cádiz) (Bourgeau, PI. Esp., 1849, núm 501a, G.); Puerto de Santa María (Cádiz) (?, G.)".

Lectotypus (designado aquí): "Periballia minuta (Loefl) Asch. et Gr. / var. baetica (Willk) // El Pedroso (Sevilla) // IV-1915 // Leg. R. Gonzalez Fragoso det. C. Vicioso" [etiqueta mecanografiada] (MA 7444, ejemplar marcado con el número 1). 


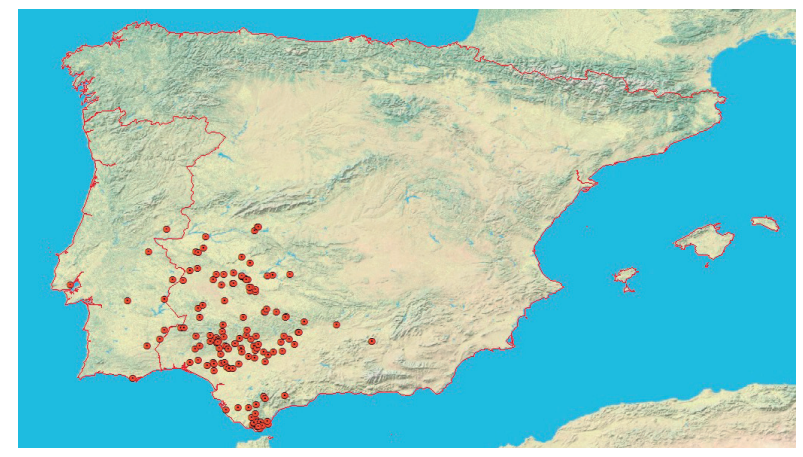

Figura 4. Mapa de distibución de Molineriella australis en la Península Ibérica. Figure 4. Distribution map of Molineriella australis in the Iberian Peninsula.

Lleva dos etiquetas de revisión del Jardín Botánico de Madrid. La más antigua como "Periballia minuta (L.) Asch. \& Graebn. ssp. australis Paun." manuscrita por E. Paunero en 1955; la más reciente como "Molineria australis Paun.", de 1962, sin firma pero con la misma letra de E. Paunero.

Isolectotipi. El pliego MA 7444 contiene otros seis ejemplares de $12-20 \mathrm{~cm}$; lema con callo provisto de pelos largos y ondulados, que sobrepasan la mitad del lema; anteras de c. $1 \mathrm{~mm}$.

Paralectotipi: Badajoz. Badajoz, V-1923, Sennen (MA 7443); Esparragosa de Lares, 18-IV1943, Gz. Guerrero (MA 7442). Cádiz. c. Chiclana, 5-IV-1925, Gros (MA 7445); Gibraltar, sin fecha, Clemente (MA 7454); Medina Sidonia, Bourgeau, PI. Esp., 1849, núm 501a (G 35076). Córdoba. Córdoba, R. Sagredo, 1934 (MA 7447). Sevilla. Dos Hermanas, 21-IV-1885, [¿G. Fragoso?] ex Caroli Pau herbarium hispanicum (MA 7446).

Ilustraciones: J. A. Devesa, Las Gramíneas de Extremadura: 127 lám. XXVII c-d (1991) [sub. M. minuta subsp. australis]; E. Paunero in Anales Inst. Bot. Cavanilles 14: 228 figs. b, j, I, n (1957) [sub Periballia minuta subsp. australis]; B. Valdés \& al., FI. Andalucía Occid. 3: 329 (1987) [sub Molineriella minuta subsp. australis]; fig. 2b.

Hierba anual, de 9-48 cm, uni- o multicaule. Tallos de 7-43 cm de longitud y 0,5-1 $\mathrm{mm}$ de diámetro, lisos o ligeramente acostillados. Hojas glabras, con vaina acostillada; lígula subaguda, la de las hojas inferiores $0,6-2 \mathrm{~mm}$, la de las hojas superiores 0,7-3 mm; limbo $14-150 \times 0,6-3 \mathrm{~mm}$, plano o enrrollado. Inflorescencia $1,2-7 \mathrm{~cm}$, hasta con 4 órdenes de ramificación. Espiguillas 1,21,8 mm; pedúnculo 1-5 mm. Glumas subiguales o ligeramente desiguales, ovadas o elípticas, subobtusas, algo aculeoladas en la quilla, con la parte central verdosa, anchamente membranáceas; la inferior 1-1,7 mm, la superior 1,1-1,8 mm. Artejo de la raquilla de c. 0,2 mm, glabro. Lema 1-1,8 $\mathrm{mm}$, ovado o elíptico, sin arista, con 5-7 nervios prominentes y escabriúsculos, variegado o de un pardo amarillento; callo 0,1-0,2 mm, orbicular, obtuso, con pelos de $(0,2) 0,4-1 \mathrm{~mm}$, hasta $1 / 2 \mathrm{de}$ la longitud del lema, ondulados. Pálea 1-1,4 mm, algo más corta que el lema en general. Lodículas $0,3-0,4 \mathrm{~mm}$. Anteras 0,7-1 mm. Fruto 0,8-1,1 ×0,3$0,5 \mathrm{~mm}$.

Número cromosómico: $n=4$ (Devesa et al., 1990b); 8 (Romero-Zarco, 1988).

\section{Ecología, fenología y distribución}

Pastizales efímeros, principalmente en substratos arenosos de carácter ácido; 0-1.000 m s.n.m. III-IV(VI). NW de África (Marruecos) y cuadrante SW de la Península Ibérica (fig. 4).

Observaciones. Los recuentos cromosómicos conocidos parecen indicar la existencia de plantas diplodes (Devesa et al., 1990b con material de Badajoz) y tetraploides (Romero-Zarco, $1988 \mathrm{con}$ material de Huelva), y confirman el número básico de cromosomas $x=4$ para este género (Albers, 1980 sub Periballia).

Selección del material estudiado

ESPAÑA. Badajoz. Badajoz, V-1923, Sennen (MA 7443). Esparragosa de Lares, 18-IV-1943, Gz. Guerrero (MA 7442). Esparragosa de La Serena, V-1982, S. Castroviejo (MA 289011). Fregenal de la Sierra, Zafra, 24-III-1988, R. Auriault (MA 498372). La Haba, 17-III1988, R. Tormo \& T. Ruiz (JACA 428493). Cáceres. Aliseda, Sierra del Aljibe, 29-III-1997, B. Rocher \& J.X. Soler (MA 590279). Belvis de Monroy, 2-IV-1980, E. Rico (MA 289266). Navalmoral de la Mata, 8-VII-1975, E. Valdés Bermejo (MA 548332). Cádiz. c. Chiclana, 5-IV-1925, Gros (MA 7445). Choza de las Monjas, Los Barrios, 14-III-1980, J. Arroyo et al. (SEV 73731). Cortijo de Herrambrosa, Los Barrios, 12-III-1978, B. Molesworth Allen 10521 (SEV 97895). Gibraltar, sin fecha, Clemente (MA 7454). Medina Sidonia, 21-III, E. Bourgeau (G 00035076). Córdoba. Córdoba, R. Sagredo, 1934 (MA 7447). Espiel, Puerto de Espiel, 17-IV-1978, E. Domínguez Vilches et al. (SEV 109931). Fuente Obejuna, 28-IV-1979, E. Fernández Galiano et al. (SEV 109933). Fuente Palmera, 1-V-1955, S. Rivas \& E. Fernández Galiano (MA 176307). Huelva. Entre Santa Olalla y El Ronquillo, 29-III-1986, P. Gibbs et al. (JACA 611386). Entre Valdeflores e Higuera de la Sierra, Arroyo de la Fuente del Rey, 24-II-1973, J. Rivera (MA 247320). Higuera de la Sierra, 14-IV-1978, J. Rivera (SEV 49890). Jaén. Andújar (MA 449546). Hotel Playa, Bélmez, 24III-1978, J. Pastor (MGC 21939). Málaga. Cortes de la Frontera, Arroyo de la Sauceda, 3-V-1975, B. Díez (MGC 2543). Montejaque, Los Quejigales, 5-V-1991, M. Osorio (MGC 33673). Sevilla. Entre Carmona y Lora del Río, 10IV-1976, M. Ladero \& S. Rivas Goday (MA 232756). Dos Hermanas, 21-IV-1885, [¿G. Fragoso?] ex Caroli Pau herbarium hispanicum (MA 7446). Entre Las Pajanosas y Venta del Alto, 16-IV-1972, E. Fernández Galiano et al. (SEV 100994). La Luisiana, 24-III-1973, E. Fernández Galiano \& E. Domínguez Vilches (SEV 100995).

PORTUGAL. Algarve. Circa Faro, II, sin recolector 
(MA7464). Alto Alentejo. Elvas, Herdade da Cascalheira, 1-VI-1968, Joao des Santos Simoes (LISI 53784/1999). Évora, Alandroal, Capelins (Santo António), 27-III-2001, A. Rosselló-Graell \& J. Brehm (LISU 187807). Vila Verde de Ficalho, III-1880, J. Daveau (LISU 4496). Baixo Alentejo. Mértola, 16-IV-1957, A. R. Pinto da Silva et al. (LISE 86331). Beira Baixa. Idanha-a-nova, Ladoeiro, Pomar do Sr. Barata, 15-IV-1980, J. M. Martins (LISI 53786/1999); íbidem, Quinta da Malhada, 15-IV-1980, J. M. Martins (LISI 53785/1999). Estremadura. Sacavém, en tierras cultivadas de Quinta de Aldeia (E.A.N.), 7-V1942, Fontes \& M. Silva (LISE 8897).

\section{M. laevis (Brot.) Rouy, FI. Fr. 14: 103 (1913)}

Aira laevis Brot., Fl. Lusit. 1: 90 (1804) [basiónimo] $\equiv$ Molineria leavis (Brot.) Hack., Cat. Rais. Gramin. Portugal 17 (1800)

三 Periballia laevis (Brot.) Asch. \& Graebn. Syn. Mitteleur. Fl. 2(1): 297 (1899)

Indicación locotípica: "Hab. In montibus aridis frigidis, circa Conimbricam et alibi in Beira".

Neotypus (designado aquí): "Flora Lusitanica (Soc. Brot. 2. ${ }^{\circ}$ anno) // 166, Aira lendigera Lag. / Aira laevis Brot. // Perto de Coimbra (Santo Antonio dos Olivaes / Colh. A. de Castro Freire. / abril de 1881", LISU P 4514, ejemplar marcado con el $n^{\circ} 1$. Isoneotypi: LISU P 4514 (10 ejemplares restantes).

Hierba anual, de (3)4,5-45 cm, uni- o multicaule. Tallos de $(2,5) 5-38 \mathrm{~cm}$ de longitud y de 0,2-1 mm de diámetro, lisos o ligeramente acostillados. Hojas glabras, con vaina acostillada; lígula subaguda, la de las hojas inferiores 0,4-3,2 $\mathrm{mm}$, la de las hojas superiores 0,7-3 mm; limbo de 6-85 × 0,3-1,6 mm, plano o enrrollado. Inflorescencia $0,5-7 \mathrm{~cm}$, con 2-6 órdenes de ramificación. Espiguillas 1,4-2,5(3) $\mathrm{mm}$; pedúnculo 1-12 mm. Glumas subiguales 0 ligeramente desiguales, oblongo-lanceoladas, subagudas, algo aculeoladas en la quilla, con la parte central verdosa, anchamente membranáceas; la inferior 1,2-2,2(2,4) mm, la superior 1,2-2,3(2,6) $\mathrm{mm}$. Artejo de la raquilla entre las dos flores $0,2-$ 0,3 mm, glabro. Lema 1,2-2(2,5) mm, oblongo, escabriúsculo en 1/2 inferior, variegado, con la parte inferior verdosa y la superior de un pardo amarillento, con 5 nervios escabriúsculos, casi siempre aristado, excepcionalmente sin arista; callo 0,2-0,3 mm, orbicular, obtuso, con pelos de $0,1-0,5 \mathrm{~mm}$, rectos; arista $(0,1) 0,8-2(2,5) \mathrm{mm}$, recta o flexionada, inserta en 1/4-1/3 superior del dorso, generalmente sobrepasando el ápice del lema, excepcionalmente ausente. Pálea 1,1-1,7(2,2) mm, algo más corta que el lema. Lodículas 0,3-0,4 mm. Anteras 0,6-1,4 mm. Fruto 1-1,5 × 0,3-0,6 mm.

Observaciones. - No se conoce la existencia de material original de esta especie en la colección de Brotero (LISU) ni en la de Valorado (cf. Coutinho, 1916), por lo que con toda probabilidad no se

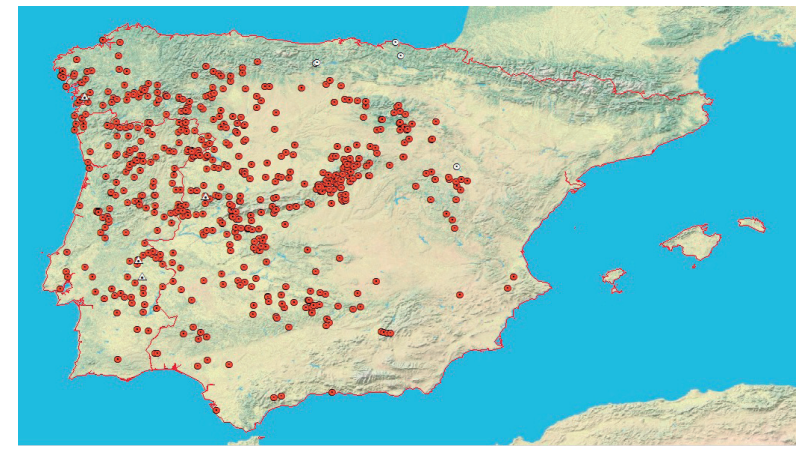

Figura 5. Mapa de distibución de Molineriella laevis en la Península Ibérica: material estudiado de $M$. laevis var. laevis (círculos oscuros) y localidades adicionales tomadas de la bibliografía (círculos claros); material estudiado de M. laevis var. submutica (triángulos claros). Figure 5. Distribution map of Molineriella laevis in the Iberian Peninsula: studied material of $\mathrm{M}$. laevis var. laevis (dark circles), and additional localities from the bibliography (white circles); studied material of M. laevis var. submutica (white triangles).

conserva en la actualidad, siendo necesaria la designación de un neotipo, que se ha seleccionado entre el material histórico estudiado procedente de los alrededores de Coimbra, la localidad clásica. En el territorio hay dos pautas de variación con respecto a la presencia y tamaño de la arista. Una de ellas, la integran plantas con arista del lema exerta, claramente visible, carácter coincidente con la variabilidad señalada por Brotero. Todas las plantas estudiadas procedentes de la zona de la localidad clásica presentan aristas claramente visibles de 0,7-1,8 mm. Estas plantas constituyen la var. laevis, muy frecuente y de amplia distribución. Por otro lado, algunos ejemplares del oeste peninsular tienen lema sin arista o con arista casi invisible, pues es corta, fina y no sobrepasa el ápice. Estas plantas, que se pueden confundir con M. australis por el tamaño de sus anteras, difieren no obstante por sus lemas oblongos (no obovados o elípticos como en las otras dos especies) y por su callo con pelos relativamente cortos y rectos. Se proponen aquí integrantes de una nueva variedad, var. submutica.

\section{Clave para las variedades (fig. 2)}

1. Lema con arista de $0,8-2(2,5) \mathrm{mm}$, que sobrepasa claramente el ápice ........................ 3a. var. laevis

- Lema sin arista, o con arista de 0,1-0,6 mm, que no sobrepasa al ápice

3b. var. submutica

3a. var. laevis

$=$ Aira lendigera Lag. in Var. Cien. Lit. Art. 4(19): 
39 (1805); Gen. Sp. Nov. 3 (1816)

Ilustraciones: J. A. Devesa, Las Gramíneas de Extremadura: 127 lám. XXVII e-f (1991); E. Paunero in Anales Inst. Bot. Cavanilles 14: 229 (1957) [sub Periballia laevis]; B. Valdés \& al., FI. Andalucía Occid. 3: 330 (1987); fig. 2d.

Hierba de (3)4,5-45 cm. Espiguillas 1,4-2,5(3) $\mathrm{mm}$. Gluma inferior $1,2-2,2(2,4) \mathrm{mm}$, la superior 1,2-2,3(2,6) mm. Lema 1,2-2(2,5) $\mathrm{mm}$, con arista de $0,8-2(2,5) \mathrm{mm}$, claramente visible y que sobrepasa el ápice, por lo general sobresaliendo por encima de las glumas. Anteras 0,6-1,4 mm.

Número cromosómico: $n=4$ (Devesa et al., 1990a); $2 n=8$ (Fernandes \& Queirós, 1969).

Ecología, fenología y distribución

Pastizales efímeros, principalmente en substratos arenosos de carácter ácido; 0-1800 m s.n.m. III-VII(VIII). NW de África (Marruecos) y gran parte de la Península Ibérica, salvo el NE, siendo rara en el extremo $\mathrm{N}$ (fig. 5).

Observaciones. Ni en la colección de A. J. Cavanilles (Garilleti, 1993) ni entre los materiales de M. Lagasca estudiados por Fernández Casas \& Gamarra (1993), se mencionan ejemplares identificados como Aira lendigera. No obstante, la identidad de Aira lendigera se ha podido confirmar mediante el examen de varios pliegos antiguos conservados en el Herbario del Jardín Botánico de Madrid (MA 7374; 7383; 185198). Los dos primeros llevan etiqueta manuscrita de Lagasca; pero ninguno reune todos los requisitos necesarios para ser designado como lectotipo. En el Herbario Gaspar Xuárez de la Universidad de Buenos Aires hay un pliego similar a estos (BAA 00000034 , foto!) procedente del herbario personal del agrostólogo L. R. Parodi, quien lo obtuvo en mayo de 1935 en Madrid, según consta en una de sus etiquetas, donde él mismo escribió: "Probablemente es el tipo Sin otros datos en la etiqueta". Todos los ejemplares contenidos en estos pliegos se identifican con $M$. laevis. Los números cromosómicos gamético $\mathrm{n}=4$ (Devesa et al., 1990a con material de Cáceres) y esporofítico $2 n=8$ (Fernandes \& Queirós, 1969 con material de Portugal) están en consonancia con el número básico de cromosomas $x=7$ confirmado para otras especies del género. En el sitio web Chromosome Counts Database, version 1.45 <http://ccdb.tau.ac.il/home/> (consultado el 25 de septiembre de 2017) se recogen varios recuentos $\mathrm{n}=7$ y $2 \mathrm{n}=14$ para esta especie, números que difieren notablemente de los publicados para plantas ibéricas. Sin embargo, estos recuentos fueron publicados originalmente bajo el binomio Aira elegans Willd. ex Gaudin, un nombre de dudosa validez que en algunas fuentes figura entre los sinónimos de Periballia laevis (p. ej. en el sitio web de The Plant List <http://www.theplantlist. org> (consultado el 25 de septiembre de 2017). En la descripción asociada a este binomio se lee: "spiculis quadruplo mininoribus [con respecto a Aira cariophyllea $\mathrm{L}$. que se describe en la página anterior]... flosculo quidem utroque sessili, sed altero superiore aristato. altero inferiore mutico" (Gaudin, 1811: 130). Esta descripción podría corresponder a alguna especie del género Aira de espiguillas pequeñas, que con frecuencia pueden presentar una sola flor aristada, como Aira tenorei Guss. o A. elegantissima Schur, pero no a Molineriella laevis; por tanto, dichos recuentos podrían ser atribuidos a alguna especie del género Aira. A las provincias que se relacionan a continuación en el material seleccionado, hay que añadir las de Cantabria (Aedo et al., 1993; Laínz, 1957), Vizcaya (Willkomm, 1861) y Zaragoza (Lagasca, 1816).

\section{Selección del material estudiado}

ESPAÑA. Álava. Urturi, 6-V-1990, P. UribeEchebarria (JACA 17190). Albacete. Montes de Ontur, sin fecha, E. Paunero (MA 7360). Ávila. Arenas de San Pedro, 8-V-1963, P. Montserrat (JACA 180963). Casavieja, Sierra de Gredos, 17-IV-1954, A. Rodríguez (MA 204946). Peguerinos, 24-Vl-1985, A. Galán de Mera (GDA 35043). Badajoz. Cabeza del Buey, vallicar cerca de Zarza Capilla, 1-V-1964, P. Montserrat \& E. Robres (JACA 58164). La Serena, 12-IV-1996, F. B. Navarro \& L. Baena (GDA 41717). Villar del Rey, 24-III-1975, A. Segura Zubizarreta (MA 362787). Burgos. Estépar, 12-V-1914, P. Font Quer (MA 7346). Humada, Ordejón de Arriba, 11-VI-1991, J. A. Alejandre (JACA 353794). Regumiel de la Sierra, 22-V-1923, Hnos Claudio \& Elías (G 00035051). Cáceres. Entre Cáceres y Plasencia, 15-IV-1984, C. Morales (GDA 20899). Miravete, 15-IV1989, P. Montserrat (JACA 33189). Serrejón, 17-V-1985, C. Morales (GDA 23562). Cádiz. Chiclana, sin fecha, Clemente (MA 7353). Ciudad Real. Fuencaliente, 10V-1997, P. Montserrat (JACA 50097). Puertollano, Valle de la Alcudia, 26-Vl-1983, G. Blanca et al. (GDA 20908). Santa Cruz de Mudela, V-1958, E. Paunero (MA 289516). Córdoba. Cardeña, finca de Yegüerizo, 30-V-1976, J. A. Devesa (SEV 34915). Cardeña-Fuencaliente, 7-VI1963, P. Montserrat \& N. Y. Sandwith (JACA 47063). Río Yeguas, Sierra Morena, 20-IV-1993, M. Melendo (GDA 41183). Cuenca. Cañete, 24-V-1974, A. González \& G. López (MA 445534). Guadalajara. El Pedregal, 1-VI1981, D. Gómez \& G. Montserrat (JACA 554781). Puebla de Beleña, Laguna Grande, 31-V-1986, P. Pascual \& M. Ventureira (MA 401957). Rebollosa de Jadraque, 19V-1968, A. Segura Zubizarreta (MA 362819). Huelva. Almonte, 1-V-1931, E. Paunero (MA 7355). Entre El Granado y Villanueva de los Castillejos, 11-III-1977, B. Cabezudo \& S. Silvestre (SEV 126822). Hinojales, 20-V-1943, C. Vicioso (MA 7354). Jaén. Andújar, Coto Medianerías, 22-IV-2003, M. Mañani \& R. Porras (FCO 29002). Santa Elena, VI-1961, sin recolector (GDA 30837). Sierra de Cazorla, VII-1980, J. Mansanet \& R. 
Currás (MA 463127). La Coruña. Esclavitud, 6-IV-1946, Bellot (SANT 6052). San Cibrán, Carnota, 25-III-1995, R. S. Louzán (MA 581184). Santiago de Compostela, sin fecha, P. Romero et al. (SEV 102226). La Rioja. El Rasillo de Cameros, 20-VI, I. Zubia (MA 7336). Nieva de Cameros, sin fecha, E. Paunero (MA 7337). León. La Cepeda, 2-V-2003, C. Pérez Morales (LEB 103541). Puerto de Manzanal (BC 649232). San Isidro, 26-IV, E. Bourgeau (MA 7314). Valdemora, unos 4,5 km al este, 21V-2013, F. Gómiz (BC 879952). Lugo. Moreda, Monforte de Lemos, 30-III-1989, M. Buján \& M. I. Romero (SANT 22682). Ribas do Sil, A Cubela, 27-IV-1996, J. Amigo et al. (SANT 34666). Touza, Oleiros, Carballedo, 24-III1989, M. I. Romero \& M. Buján (SANT 22669). Madrid. El Escorial, 25-V-1877, Torrepando (G 00035055). Madrid, sin fecha, Cavanilles (MA 7374); íbidem, sin fecha, Lagasca (MA 7383); íbidem, sin fecha ni recolector (BAA 00000034 , foto!). Puerto de la Morcuera-Rascafría, 23V-1968, P. Montserrat (JACA 162568). Torrelaguna, VII, VII, sin recolector, ex Instituto de Enseñanza Secundaria San Isidro (MA 573594). Málaga. Nava de San Luis, Sierra de las Nieves, 6-VII-2005, C. Aedo \& A. Galán de Mera (MA 730150). Torrox, V-1908, Domingo (G 00035049). Orense. A Rua, 30-III-1988, M. I. Romero \& M. Bujan (JACA 439993). Cabeza de Manzaneda, 26-VI1898, M. Gandoger (MA 7316). Monte Faro, 12-V-1954, A. Rodríguez (MA 200338). Palencia. Entre Saldaña y Herrera de Pisuerga (MA 723513). Pontevedra. Lérez, 19-IV-1950, A. Rodríguez (MA 200343). Lourizán, 4-V1951, A. Rodríguez (MA 200344). Redondela, 19-IV1984, C. Morales (GDA 20900). Salamanca. La Encina de San Silvestre, 13-V-1975, A. Soler (SEV 103484). Serradilla del Llano, 24-VI-1984, E. Rico (MA 560848). Villarino de los Aires, 27-IV-1977, J. Sánchez (GDA 8134). Segovia. Aldeonsancho, Cantalejo, 15-V-1983, T. Romero (SEV 121868). El Espinar, 19-VII-1957, A. Rodríguez (MA 181372). Fresno de la Fuente, 29-VI1984, M. J. Illueca (MA 308896). Sevilla. Carrión de los Céspedes, 26-III-1968, A. Segura Zubizarreta (MA 362785). Soria. Carbonera de Frentes, 20-V-1989, A. Segura Zubizarreta (JACA 278396; MA580402). Reznos, 19-VI-1935, C. Vicioso (MA 7341). Teruel. Blancas, sin fecha, Almagro (MA 7351). Monreal del Campo, VII1894, J. Bendicto (MA 7352). Toledo. Talavera de la Reina, 24-V-1966, E. Paunero \& E. Fernández Galiano (MA 183291). Valencia. Valle de Albaida, 3-VIII-1904, R. Prosper (MA 7361). Valladolid. Castromonte, V-1903, Hno. J. Vital (BC 951905). La Pedraja del Portillo, 1-V1987, A. R. Burgaz \& E. Saiz (MA 498988). Olmedo, 3-VII-1925, Gutiérrez (MA 7327). Zamora. Benavente, 2-VI-1971, M. Mayor (JACA 756171). San Martín de Castañeda, Laguna de los Peces, 10-VII-1979, M. Ladero (GDA 7387). Vigo de Sanabria, 18-V-1987, A. Roa (MA 509745).

Material de origen español pero sin localidad concreta ni fecha, "Se cría junto a Madrid, en Used, Castilla la vieja y Alcarria", atribuido a Lagasca (MA 185198).

PORTUGAL. Alto Alentejo. Chancelaria, Alter do Chão, 5-IV-1955, Beliz \& Abreu (LISE 48115). Évora, IV-1882, J. Daveau (G 00035061). San Cristovao, Corta Rabor de Baixo, Montemor-o-novo, 20-IV-2006, D. E.
Santo et al. (LISI 69/2010). Baixo Alentejo. Entre Corte Figueira et Mü, VI/VII-1885, J. Daveau (LISU 4505). Serpa, Herdade de Charneca, 25-IV-1952, F. G. Palma (LISI 53748/1999). Beira Alta. Caramulo, V-1892, A. Moller (MA 7322). Monteiras, Castro Daire, 24-VI-1969, J. J. Silva Melo (LISI 53766/1999). Serra da Estrela, Fraga da Cruz, VII-1882, A. R. da Cunha (LISU 4507). Beira Baixa. Covilhã, próximo de Refugio, 25-04-1962, A. Rozeira et al. (HVR 11113). Penamacor, Aldeia Joao Pires, 17-V-1987, M. Lousa et al. (LISI 53772/1999). Vale das Eiras, Salvaterra do Extremo, Idanha-anova, 26-V-1972, M.T. Vasconcelos \& M.N. Filipe (LISI 53768/1999). Beira Litoral. Aroes a $200 \mathrm{~m}$ de E-NE de Coro, Vale de Cambra, 4-VII-1968, A. da Cunha Direito (LISI 53765/1999). Coimbra, V-1901, M. Ferreira (MA 289267). Miranda do Corvo, 24-IV-1943, F. de Sousa (LISE 8038). Douro Litoral. Albergaria das Cabras, margem do rio Caima, 5-VI-1968, A. da Cunha Direito (LISI 53763/1999). Amarante, 12-IV-1953, F. Mendes et al. (LISU 65390). Vila Nova de Gaia, Grijó, IV-1888, J. A. d'Araujo Castro (G 00035059). Estremadura. Lisboa, sin fecha, Zimmermann (COI 78527). Vale da Ursa, IV1911, F.R. Palhinha \& F. Mendes (LISU 4517). Minho. Alto Borragueiro, Sierra de Gerês, 12-VIII-1948, S. Rivas Goday (MA 164207). Montedôr, Gandra, VI-1886, A.R. da Cunha (COI 78547). Viana do Castelo, Caminha, Serra do Arga, Arga de Sao Joao, Alto do Espiñeiro, 7-VI-2010, J.L. Fernández Alonso, M.B. Crespo et al. (ABH 56798). Ribatejo. Benfica do Ribatejo, Casalinho, Almeirim, 3-V1979, T. Vasconcelos et al. (LISI 53773/1999). Ferreira do Zêzere, Machiol, V-1914, M. Ferreira (COI 78530). Trás-os-Montes e Alto Douro. Bragança, Carragosa, Abessoeira, 28-V-1972, A.M. Marcos \& C. Almeida (LISI 53769/1999). Bragança, Pereiros, 24-V-1966, P. Silva \& B. Rainha (LISE 69481). Peredo da BempostaMogadouro, 4-V-1996, N. Marcos \& T. Koe (HVR 6466).

3b. Molineriella laevis var. submutica Cebrino \& Romero Zarco, var. nova

A typo differt arista breviore vel nula, (0)0,1-0,6 $\mathrm{mm}$ longa, lemma non superante.

Se diferencia del tipo por su arista más corta o nula, de (0)0,1-0,6 mm, que no sobrepasa al lema.

Hierba de 11-24 cm. Espiguillas 1,6-2 mm. Gluma inferior 1,3-1,8 mm, la superior 1,5-1,9 mm. Lema 1,3-1,6 mm, sin arista o con arista de 0,1-0,6 $\mathrm{mm}$, apenas visible, que no sobrepasa al lema ni sobresale por encima de las glumas. Anteras 0,8$0,9 \mathrm{~mm}$.

Holotypus: [Portugal, Alto Alentejo] Crato: Crato e Mártires; Tapada do Doutor; leg.: J. da Gama, 13-IV-1956 (LISI 53782/1999).

Ilustración: fig. 2c.

Número cromosómico: desconocido.

Ecología, fenología y distribución

Pastizales efímeros en terrenos esquistosos o graníticos; 200-900 m. IV-VI. Endemismo del oeste la Península Ibérica (fig. 5).

Observaciones.--No se han observado otras diferencias que puedan apoyar una categoría 
subespecífica para estas plantas, aunque al ser menos frecuentes su variabilidad es menor que en la variedad típica. El epíteto submutica alude al carácter distintivo de la nueva variedad, cuyas lemas carecen de arista o llevan una arista apenas visible.

\section{Material estudiado}

ESPAÑA. Pontevedra. Lourizán 14-IV-1960, A. Rodríguez (MA 441960, ejemplar marcado con el $n^{\circ} 1$ ). Salamanca. Serradilla del Llano, 24-VI-1984, E. Rico (FCO 25138).

PORTUGAL. Alto Alentejo. Crato, Crato e Mártires, Tapada do Doutor, 13-IV-1956, Joao da Gama Matutino (LISI 53782/1999). Souzel, Santo Amaro, junto a la entrada de Santo Amaro a Souzel, 23-IV-1958, Cláudio Pereira Godinho (LISI 53783/1999). Freg. Vale do Peso, Couto do Capão, 14-IV-1956, Joao da Gama Matutino (LISI 53755/1999, planta B).

\section{Insertae sedis}

Aira glabrata Brot. Fl. Lusit. 1: 91 (1804)

De acuerdo con Hackel (1880: 17) dos de las especies descritas por Brotero (1804, 1: 90-94) corresponden a variaciones de una misma especie. Las indicaciones locotípicas son casi idénticas:

Aira laevis Brot:: "Hab. in montibus aridis frigidis, circa Conimbricam et alibi in Beira."

Aira glabrata Brot.: "Hab. in montosis glareosis, frigidis circa Conimbricam, et alibi in Beira boreali."

Hackel (I.c.), utiliza como nombre prioritario Aira laevis, descrita en primer lugar en la página 90, y reduce al rango varietal a $A$. glabrata, descrita en la página 91 . Este último nombre ha sido poco usado por los autores del siglo XIX y, en la actualidad, está casi olvidado.

De acuerdo con las descripciones que da el mismo Brotero de ambas especies, la principal diferencia entre ellas sería:

Aira laevis: "...pedicello minimo, leviter ad lentem villoso, impositus. Corolla valvula exterior glabra, ad lentemm inferne vix pubescens..."[artejo de la raquilla corto, ligeramente viloso bajo la lupa. Lema glabro, bajo la lupa apenas pubescente en la parte inferior]

Aira glabrata: "...pedicello glabro; corolla válvula usque ad basin glaberrima..." [artejo de la raquilla glabro; lema muy glabra hasta la base].

Los pelos del artejo de la raquilla y del callo de las flores son cortos (c. 0,2 mm) y generalmente escasos en todo el material estudiado. En las plantas del género Molineriella procedente de los alrededores de Coimbra, no se ha detectado ninguna muestra que responda plenamente a la descripción de Aira glabrata. Solo se han encontrado plantas que responden a la descripción de Aira laevis.
Por otra parte, algunos aspectos de la descripción de Aira grabrata no se corresponden con la identidad que le atribuye Hackel (I.c.). Así, la descripción de la inflorescencia: "Panicula ovata, laxe patens... ante et post florescentiam contracta" no se puede aplicar a las inflorescencias de Aira laevis, especie que mantiene la panícula abierta y laxa después de la fructificación y durante la dispersión de los frutos. Además, la aclaración que hace el propio Brotero (op. c.: 92) sobre la afinidad de Aira glabrata no arroja mucha luz sobre su identidad sino que, muy al contrario, la oscurece: "Affinis A. spicatae; sed laxe patens pedunculi non villosi, sed glaberrimi; arista paulo infra apicem corolla sita, nec tortilis". Aira spicata L. es el basiónimo de Trisetum spicatum (L.) K. Richt., especie propia de los prados alpinos que obviamente no figura en la Flora Lusitanica de Brotero.

Por consiguiente, se considera aquí que Aira glabrata Brot. es un nombre de aplicación dudosa, aunque según los autores portugueses podría corresponder a un taxón infraespecífico dentro de Aira laevis Brot.

\section{Agradecimientos}

Las micrografías al microscopio electrónico se realizaron en el Servicio de Herbario de la Universidad de Sevilla (personal técnico: M. J. Ariza). Al Dr. Francisco Javier Salgueiro por ocuparse de los préstamos de materiales de diversos centros. A Pilar Fernández Piedra por la búsqueda bibliográfica. A Rodrigo Tavera, colaborador habitual de Flora iberica, le agradecemos sus observaciones y el diseño de la figura 2. Se agradece a los responsables de los herbarios citados el préstamo de sus materiales. Los doctores J. A. Devesa y S. Talavera sugirieron importantes cambios que han mejorado notablemente el texto.

\section{Referencias}

Aedo, C., Aldasoro, J. J., Argüelles, J. M., Díaz Alonso, J. L., González, J. M., Herra, C., Laínz, M., Moreno, G., Patallo, J., \& Sánchez, O. (1993). Contribuciones al conocimiento de la flora cantábrica, Fontqueria, 36, 349-374.

Afzal-Rafii Z. \& Zevaco, C. (1974). Contribution l'tude cytotaxonomique de quelques Aireae de Corse et de Sardaigne.. Bull. Soc. Bot. France, 121(7-8), 281-286.

Albers, F. (1980). Comparative karyology of the grass subtribes Aristaveninae and Airinae (Poaceae-Avenae). Pl. Syst. Evol., 136: 137-167

ArcGIS9 (2009). ArcGIS ArcView Version 9.3.1. Redlands, CA: ESRI (Environmental Systems Research Institute Inc.).

Ascherson, P. F. \& Graebner, K. O. (1899). Synopsis der mitteleuropäischen Flora, 2(1). Leipzig. 
Bolòs, O. \& Vigo, J. (2001) Flora dels Països Catalans. Editorial Barcino, Barcelona.

Brotero, F. A. (1804). Flora Lusitanica. Lisboa.

Burdet, H. M., Charpin, A. \& Jacquemoud, H. (1981). Types nomenclaturaux des taxa ibériques décrits par Boissier ou Reuter. 1. Gymnospermes à Graminées. Candollea, 36, 543-584.

Clayton, W. D. \& Renvoize, S. A. (1986). Genera graminum: grasses of the world. Her Majesty's Stationary Office, London.

Coutinho, A. X. P. (1916). Plantas portuguesas dos Herbarios de Brotero e de Valorado existentes na Universidade de Lisboa. Arquivos Univ. Lisboa, 3, 333-379.

Devesa, J. A. (1991). Las Gramíneas de Extremadura. Servicio de Publicaciones de la Universidad de Extremadura, Badajoz.

Devesa, J. A. (1995). Flora y Vegetación de Extremadura. Universitas Editorial. Badajoz.

Devesa, J. A., Ruiz, T., Ortega, A., Carrasco, J. P., Viera, M. C., Torno, R. \& Pastor, J. (1990a). Contribución al conocimiento cariológico de las Poaceae en Extremadura (España) I. Bol. Soc. Brot., sér. 2, 63, 29-66.

Devesa, J. A., Ruiz, T., Tormo, R., Muñoz, A., Viera, M. C., Carrasco, J. P., Ortega, A. \& Pastor, J. (1990b). Contribución al conocimiemto cariológico de las Poaceae en Extremadura (España). II. Bol. Soc. Brot., ser. 2, 64, 153-205.

Döring, E. (2009). Molekulare Phylogenie der HaferGräser (Poaceae: Pooideae: Aveneae). Tesis doctoral de la Universidad de Halle (Alemania). Publicada en Internet: <http://digital.bibliothek.uni-halle.de/hs/ content/titleinfo/177571> [consultado el 29 de marzo de 2016].

Fernandes, A. \& Queirós, M. (1969). Contribution la connaissance cytotaxinomique des spermatophyta du Portugal. I. Gramineae. Bol. Soc. Brot., ser. 2, 43, 20140

Fernández Casas, J. \& Gamarra, R. (1993). Herbarium Lagascanum. Fontqueria, 36, 67-108.

Garilleti, R. (1993). Herbarium cavanillesianumseu enumeratio plantarum exsiccatarum aliquo modo ad novitates cavanillesianas pertinentium, quae in Horti Regii Matritensis atque Londinensis Societatis Linnaecanae herbariis asservantur. Fontqueria, 38, 1-249.

Gaudin, J. F. A. P. (1811). Agrostologia Helvetica. Paris y Ginebra.

Hackel, E. (1877). Ueber einige Gräser Spaniens und Portugals. Oesterr. Bot. Z., 27,118-125.

Hackel, E. (1880). Cataloge raisonné das gramineés du Portugal. Imprimerie de l'Université. Coimbra.

Kozhuharov, S. I. \& Petrova, A. V. (1991). Chromosome numbers of Bulgarian angiosperms. Fitologija, 39, 72-77.

Lagasca, M. (1816). Genera et species plantarum, quae aut novae sunt aut nondum recte cognoscuntur. Madrid.

Laínz, M. (1957). Aportaciones al conocimiento de la Flora Cántabro-Astur. II, Collect. Bot. (Barcelona), 5, 429-460.
Linneo, C. (1753). Species Plantarum. Stockholm.

Linneo, C. (1754). Genera Plantarum, ed. 5. Stockholm. Parlatore, G. (1848-1850). Flora italiana 1. Firenze.

Pastor, J. (1992). Atlas cromosómico de la flora vascular de Andalucía occidental. Universidad de Sevilla.

Paunero, E. (1957). Las Aveneas españolas II. Anales Inst. Bot. Cavanilles, 187-251.

Paunero, E. (1964). Notas sobre gramíneas. I. Consideraciones sobre los géneros Periballia y Molineria. Anales Inst. Bot. Cavanilles, 21, 341-356.

Quintanar, A., Castroviejo, S. \& Catalán, P. (2007). Phylogeny of the tribe Aveneae -Poideae, Poaceae) inferred from plastid TRNT-F and nuclear ITS sequences. American J. Bot., 94(9), 1554-1569.

Romero, A. T. (2011). Molineriella Rouy; Periballia Trin. In: G. Blanca, B. Cabezudo, M. Cueto, C. M. Torres \& C. SalazaR (eds.), Flora Vascular de Andalucía Oriental, ed. 2: 347-348. Universidades de Almería, Granada, Jaén y Málaga, Granada.

Romero-Zarco, C. (1987). Molineriella Rouy; Periballia Trin. In: B. Valdés, S. Talavera \& E. F. Galiano (eds.) Flora Vascular de Andalucía Occidental, 3, 328-330. Ketres Ed. S.A., Barcelona.

Romero-Zarco, C. (1988). Números cromosómicos para la flora española. Números 516-527. Lagascalia, 15(1), 119-124.

Rouy, G. (1913). Flore de France 14. París.

Segura, A., Mateo Sanz, G. \& Benito Alonso, J. L. (1998). Catálogo Florístico de la Provincia de Soria. Monografías de Flora Montiberica ${ }^{\circ} 4$, Valencia.

Seijas, E. (1952). Contribución al catálogo de la Flora de Lugo. Trab. Jard. Bot. Santiago, 6, 31-62.

Serra, L., Oltra, J. E., Conca, A., Soler, J. X. \& Nebot, J. R. (2012). Catálogo de la flora del Parque Natural de la Sierra de Mariola (Alicante-Valencia). Fl. Montiber., 51, 97-125.

Soreng, R. J., Davidse, G., Peterson, P. M., Zuloaga, F. O., Judziewicz, E. J., Filgueiras, T. S., Morrone, O. \& Romaschenko, K. (2010-2014). A World-wide Phylogenetic Classification of Poaceae (Gramineae). Versión 5/04/2014<http://www.tropicos.org/project/ cnwg $>$ [consultado el 28 de abril de 2017].

Thiers, B. [continuously updated]. Index Herbariorum: A global directory of public herbaria and associated staff. New York Botanical Garden's Virtual Herbarium $<$ http://sweetgum.nybg.org/ih/> [consultado el 28 de abril de 2017]

Tison, J.-M. \& De Foucault, B. (2014). Flora Gallica. Biotope, Mèze.

Trinius, C. B. von (1820). Fundamenta Agrostographiae. Viena.

Tutin, T. G. (1980). Molineriella Rouy; Periballia Trin. In: T. G. Tutin, V. H. Heywood, N. A. Burges, D. M. Moore, D. H. Valentine, S. M. Walters And D. A. Webb. (eds.) Flora Europaea, vol. 5: 228. Cambridge, U. K.: Cambridge University Press.

Watson, L. \& Dallwitz, M. J. (1992). The grass genera of the world (Revised ed.). Oxon CAB International, Wallingford, UK.

Willkomm, H. M. (1861). Gramineae Juss. In: Willkomm \& J. M. CH. Lange, Prodromus Florae Hiapanicae, 1, 33-118. Stuttgart. 